\title{
Effective information transfer of sport skills through modeling.
}

\begin{abstract}
Visual demonstration has long been regarded as a critical instructional methodology for children's motor skill and social development. Despite its widespread importance, skills demonstrations have often been characterized by information overload, lack of clarify and an overall failure to consider differences in children's cognitive and physical abilities which play a strong role in their understanding of modeled actions. These problems result in young performers' inability to translate what they see and hear into skilled movements, and this, in turn, may have a negative effect on subsequent motivation and self-confidence. Thus, the purpose of this research is to highlight the inhibition-disinheriting effects of modeling in the physical domain, specifically effective modeling techniques for enhancing self-efficacy and motivation, and reducing anxiety and stress prevalent in high-avoidance sport skills.
\end{abstract}

Keyword: Modeling; High-avoidance sport skills. 